\title{
Método Famacha como parâmetro clínico individual de infecção por Haemonchus contortus em pequenos ruminantes
}

\author{
Famacha guide as an individual clinic parameter for Haemonchus contortus \\ infection in small ruminants
}

\author{
Marcelo Beltrão Molento ${ }^{1}$ Cláudio Tasca² Alencar Gallo $^{2}$ \\ Márcio Ferreira ${ }^{2}$ Ronaldo Bononi ${ }^{2}$ Evandro Stecca ${ }^{2}$
}

\section{RESUMO}

\begin{abstract}
O objetivo deste trabalho foi avaliar a utilização do método Famacha como estratégia auxiliar no controle do parasita Haemonchus contortus em ovinos e caprinos no Brasil. A principal característica deste método é a identificação de animais resistentes e resilientes no rebanho, sendo possível a seleção de animais que não necessitam receber tratamento antiparasitário. Além do acompanhamento clínico, foram determinados valores de hematócrito e ovos por grama de fezes (OPG). Ao término do experimento, foi determinada uma considerável redução $(75,6 \%)$ na utilização de medicação antiparasitária nos ovinos, quando comparado com o controle profilático de tratamento do rebanho todo em intervalos de 30 dias dos anos anteriores. O método Famacha, embora também indicado para uso em caprinos, requer pequenas adaptações para sua melhor aplicabilidade. O método Famacha pode ser utilizado no Brasil com o objetivo de racionalizar o uso dos compostos antiparasitários, preservando sua eficácia por períodos prolongados.
\end{abstract}

Palavras-chave: ovinos, caprinos, Haemonchus contortus, Famacha, hematócrito, OPG.

\section{ABSTRACT}

The objective of this research was to evaluate the Famacha method as an auxiliary strategy for the control of the parasite Haemonchus contortus in sheep and goats in Brazil. The main characteristic of this method is the identification of resistant and resilient animals within the flock, selecting animals that do not require drug treatment. Haematocrit levels and fecal eggs per gram
(EPG) were measure as well as the clinical examination. At the end of the experiment a considerable decrease (75.6\%) in antiparasitic drug usage was determined in sheep, when compared to the prophylactic control management, treating all flock in 30 days intervals in previous years. Although the Famacha guide is indicated to be used in goats, some particularities have to be observed to obtain better results. The Famacha method may be applicable in Brazil with the aim of reducing drug usage and prolonging its lifespan.

Key words: sheep, goats, Haemonchus contortus, Famacha, hematocrit, EPG.

\section{INTRODUÇÃO}

O parasitismo gastro-intestinal é responsável por grande parte das perdas observadas em criações de ovinos e caprinos, reduzindo o potencial produtivo destes animais, inclusive com a morte de animais jovens para reposição do plantel. O conhecimento real de tal prejuízo ainda é desconhecido, porém dentro da característica de distribuição-dispersa é possível que a maioria dos animais de um mesmo rebanho apresentem baixo grau de infecção e somente um número inferior a $20 \%$ dos animais devem conter níveis indesejáveis de infecção a ponto de causar

${ }^{1}$ Médico Veterinário, PhD, Professor de Doenças Parasitárias, Laboratório de Doenças Parasitárias, Departamento de Medicina Veterinária Preventiva, Universidade Federal de Santa Maria (UFSM), Camobi, Santa Maria, RS, 97.105-900. E-mail: molento@smail.ufsm.br

${ }^{2}$ Médico Veterinário, Autônomo. 
perdas econômicas significativas (ROBERTS \& SWAN, 1982).

Decorrente do insuficiente repasse de tecnologia ou mesmo de informações inadequadas referentes à frequiência de tratamento e a utilização correta das drogas antiparasitárias em ruminantes, foi observado uma grande diminuição da eficácia destes produtos nas principais regiões produtoras brasileiras (ECHEVARRIAet al., 1996, VIEIRA \& CAVALCANTE, 1999), com o aparecimento de cepas resistentes a vários grupos químicos, originando a resistência antihelmíntica múltipla. Pesquisadores alertam que, com a utilização de drogas de forma pouco criteriosa, muito em breve, haverá a redução das fontes de controle químico causando prejuízo para produtores que dependem desta atividade (MOLENTO \& PRICHARD, 1999; VAN WYK et al., 1999).

Estas observações vêm estimulando pesquisadores a desenvolver alternativas na busca da manutenção da eficácia das drogas antiparasitárias assim como a sustentabilidade da produção agropecuária. Tais métodos têm como objetivo central, diminuir o uso de anti-helmínticos, reduzir a concentração das drogas no meio ambiente, no leite, na carne e possibilitar a participação de produtores nos processos de certificação para melhoria da qualidade de produtos animais. Unindo todas estas possibilidades à atual busca por um mercado que prioriza a saúde, o meio ambiente e o bem-estar animal, o tratamento antiparasitário seletivo, opostamente ao tratamento profilático, pode ser uma metodologia viável para técnicos, produtores e companhias farmacêuticas.

Embora existam vários métodos laboratoriais e clínicos para diagnóstico parasitário, muitos destes são de baixa precisão. O teste mais aplicado, porém com significativa margem de variação é o que determina a quantidade de ovos por grama de fezes (OPG), realizado antes e/ou após o tratamento. Os testes de migração, eclodibilidade e desenvolvimento de larvas, hematócrito e as técnicas sorológicas (pepsinogênio, gastrina, anticorpos antiparasitos) devem ser utilizados sob condições laboratoriais, sabendo-se também de suas limitações. Entretanto, existe uma forma de se avaliar um animal/ rebanho por meio de informações que correlacionam dados clínico-laboratoriais. MALAN \& VAN WYK (1992) observaram a correlação entre a coloração da conjuntiva ocular, o valor do hematócrito e a incidência do parasita hematófago, Haemonchus contortus. VAN WYK et al. (1997) associaram os valores de hematócrito com diferentes colorações da conjuntiva ocular. Estas colorações foram preestabelecidas com auxílio de computação gráfica, representando cinco graus de anemia, incluindo pequenas variações para cada grau. Estes autores comprovaram também que os diferentes graus de anemia apresentaram correlação de 0,8 , com grau de confiabilidade superior a $95 \%$, para infecções causadas por $\boldsymbol{H}$. contortus. Foi então que estes autores apresentaram o método Famacha. O objetivo deste método é identificar clinicamente animais resistentes, resilientes e sensíveis às infecções parasitárias, otimizando o tratamento de forma seletiva em situações reais no campo, sem a necessidade de recursos laboratoriais.

BATH \& VAN WYK (2001) utilizaram o método Famacha durante o período de 1998 a 1999 avaliando 10 rebanhos em diferentes regiões da África do Sul. Estes autores observaram uma redução entre 38 e $96 \%$, com média de $58,4 \%$ na utilização e nos custos com dosificações. No Brasil, dados preliminares revelaram que, após a utilização deste método durante um período de 120 dias (março a junho de 2000), foi possível reduzir em $79,5 \%$ as aplicações com medicação antiparasitária em ovinos (MOLENTO \& DANTAS, 2001).

O objetivo deste trabalho foi avaliar a utilização do método Famacha em ovinos e caprinos, procurando identificar características próprias em relação à metodologia proposta para as duas espécies animais.

\section{MATERIAL E MÉTODOS}

\section{Animais}

Foram utilizados 37 ovinos adultos de raças não puras naturalmente infectados, sendo 31 fêmeas e seis machos, acompanhados durante um período de 180 dias (outubro de 2001 a março de 2002). O tratamento convencional com drogas antiparasitárias foi interrompido 30 dias antes do início do experimento. Todos os animais pertenciam a um rebanho único, ficando em pastagem formada de coast-cross (Cinodon dactylon) durante o período diurno, obedecendo a um esquema de rodízio de pastagens de acordo com a disponibilidade da gramínea. Os animais receberam aproximadamente $400 \mathrm{~g}$ de farelo de trigo/dia para manutenção no período da tarde após serem recolhidos para recinto coberto, elevado e com piso ripado. Os animais receberam água ad libitum em bebedouros automáticos. Quatorze caprinos mestiços (12 fêmeas e dois machos) adultos foram observados entre os meses de maio e julho de 2002. A intensidade da coloração da conjuntiva de caprinos foi comparada com ovinos para melhor visualização e treinamento dos avaliadores. As 
propriedades acompanhadas estão situadas no município de Umuarama, PR.

\section{Exame clínico}

A inspeção da conjuntiva dos animais foi realizada em triplicatas por técnicos devidamente treinados com intervalo mínimo de duas a três semanas, observado-se também a presença de edema submandibular e/ou diarréia. $\mathrm{O}$ exame foi realizado através da comparação de diferentes tonalidades, de vermelho-rosado até o branco pálido da conjuntiva, representada com os números de 1 a 5 e comparados com o cartão guia desenvolvido para utilização no campo. Os valores de hematócrito correspondentes ao grau Famacha são: 1: $28 \%$ e valores acima, 2: entre 23 e $27 \%$, 3: entre 18 e $22 \%$, 4 : entre 13 e $17 \%$ e $5: 12 \%$ e abaixo. A indicação de tratamento pelo cartão é baseada unicamente na coloração da conjuntiva e quanto maior o grau Famacha maior a urgência do tratamento.

\section{Exame laboratorial}

Para análise, interpretação e correlação dos resultados foi realizada a colheita de fezes e sangue dos animais. As fezes foram colhidas diretamente da ampola retal para contagem, cultura e identificação das larvas. Foi utilizada a técnica de McMaster modificada (GORDON \& WHITLOCK, 1939) para a determinação do OPG. A identificação das larvas infectantes foi realizada através da técnica descrita por WERTEJUK (1955). As amostras de sangue foram colhidas da veia jugular dos animais por meio de tubos BD vacutainer ${ }^{\hat{\imath}}$ (Becton \& Dickinson, Reino Unido) com anticoagulante. Após a colheita, os tubos foram enviados ao laboratório para a determinação do percentual de hematócrito. Seguindo as indicações do método Famacha, todos os animais que apresentaram hematócrito abaixo de $22 \%$ e conjuntiva com graus 3 , 4 ou 5, foram tratados com ivermectina oral (Ivomec ${ }^{\hat{a}}$ ovinos, Merial Saúde Animal, Brasil). Adicionalmente os animais que apresentaram OPG acima de 400 também receberam tratamento. A eficácia do anti-helmíntico empregado foi determinada pela fórmula:

$\mathrm{E}=\mathrm{OPG}$ antes do tratamento $-\mathrm{OPG}$ pós tratamento $\mathrm{x} 100$ OPG antes do tratamento

\section{Avaliação dos examinadores}

Como mencionado anteriormente, todas as etapas do exame clínico, em ovinos e em caprinos, foram acompanhadas por técnicos treinados. Estes técnicos também foram avaliados conferindo-se o valor predito para o grau Famacha e o percentual de hematócrito. Após o exame dos animais e análise dos resultados, o índice de acerto individual foi transformado em média e expresso em forma de tabelas.

Os dados clínico-laboratoriais foram coletados em forma de planilhas por amostragem e por animal para posterior analise da correlação.

\section{RESULTADOS E DISCUSSÃO}

A seqüência das avaliações clínicas demonstra o grau de sanidade dos animais e a frequiência de tratamentos através da coloração da conjuntiva (Figura 1). Este acompanhamento também fornece informações importantes quanto aos períodos de maior infecção parasitária no decorrer do período. A determinação criteriosa de incluir os animais em cinco categorias diferenciadas auxilia na definição de quais animais serão dosificados ou não. Com base no conjunto de observações: Famacha - hematócrito OPG, de todos os períodos, observou-se um percentual entre 10 e $63,3 \%$ de ovinos que necessitaram ser tratados. Já no primeiro exame, 36,7\% do rebanho não foram tratados, demonstrando, então, que existe uma parcela da população de hospedeiros que pode ser identificada através de exame clínico e permanecer sem tratamento. Após 180 dias, somente dois ovinos apresentaram hematócrito abaixo de $22 \%$, revelando total ausência de sinais clínicos de infecção por $\boldsymbol{H}$. contortus. Em relação à necessidade de utilização de medicação antiparasitária, 29 animais necessitaram ser tratados uma vez, cinco animais necessitaram ser tratados duas vezes e somente dois animais necessitaram ser tratados três vezes, totalizando 36 animais durante o período de avaliação. Foram efetuadas 45 dosificações de forma seletiva, o que representa uma redução de dosificações/custos na ordem de 75,6\%, quando comparado ao manejo de tratamento antiparasitário adotado no mesmo período do ano anterior (185 doses) na mesma propriedade de ovinos. Nenhum óbito foi observado durante a fase experimental.

O método Famacha apresentou uma correlação de 1:0,8 com o valor de hematócrito em ovinos. Mesmo com contagens de OPG acima de 1500 em vários animais não foram observados sinais de anemia. Este fato sugere a grande capacidade de alguns animais de poderem suportar altas cargas parasitárias. Estes animais são denominados resilientes.

$\mathrm{Na}$ coprocultura, os gêneros mais prevalentes foram: Haemonchus sp., Cooperia sp. e Trichostrongylus sp. com 60, 30 e 10\%, respectivamente. Foram encontrados também Moniezia sp. e oocistos em número variado e 


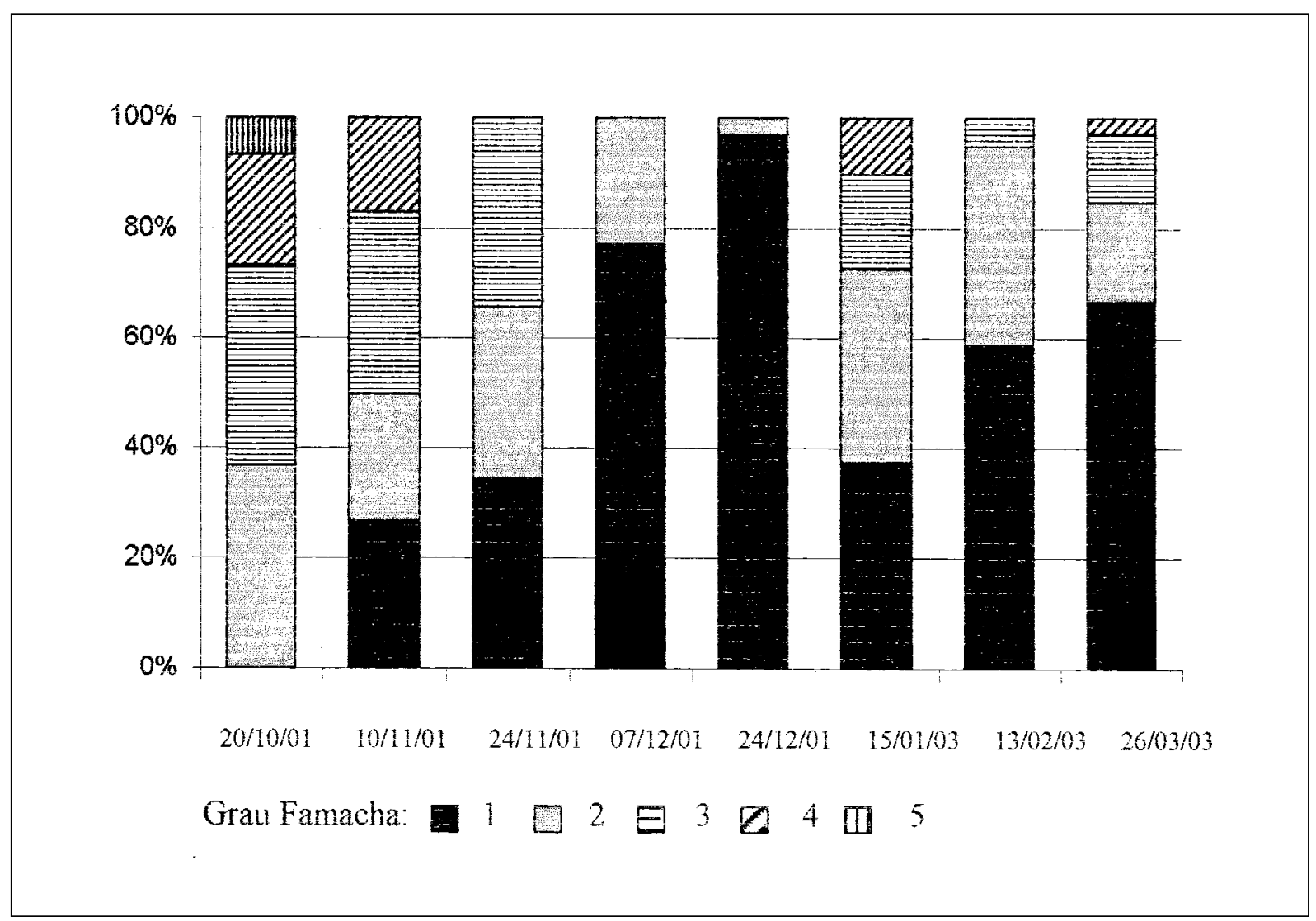

Figura 1 - Determinação dos diferentes graus Famacha entre os períodos de 20/10 de 2001 a 26/03 de 2002.

inconsistente. Os exames de OPG em ovinos determinaram que a eficácia do medicamento utilizado no início do estudo (20/10/2001) foi de $99 \%$ e ao final (13/03/2002) foi de $96 \%$ contra o $\boldsymbol{H}$. contortus, com intervalo de confiança inferior de $85 \%$.

Foi observada que a coloração da conjuntiva de caprinos sadios tem menor intensidade quando comparada a ovinos sadios. Outra observação de grande importância é que para o exame clínico, a exposição da conjuntiva deve ser feita respeitando um período de preenchimento capilar mais demorado do que em ovinos. No caso dos caprinos, ocorre melhor diferenciação da coloração da conjuntiva quando se espera em torno de 6 a 8 segundos após a exposição da conjuntiva. Neste experimento, foram identificados corretamente sete dos 11 animais (Tabela 1). De acordo com os dados obtidos, o método Famacha foi válido para os animais: $1,3,5,6,8,9,10$ e 11 , correspondendo a $73 \%$ de acerto. Destes, somente os animais 6 e 11 poderiam sofrer sérias conseqüências com o uso deste método. A pontuação dos avaliadores demonstrou padrão semelhante aos apresentados em ovinos com uma grande diminuição das variações entre o estimado pelo guia Famacha e os valores de hematócrito. O percentual de acerto só não foi maior devido ao erro na estimativa nos animais de número 2, 4 e 7 (Tabela 1).

A redução da pontuação dos valores estimados para o grau Famacha indica que os observadores adquiriram maior confiabilidade no método após cinco avaliações (Tabela 2). A pontuação, que deve ser próxima de zero, foi gradativamente sendo reduzida até atingir um nível de confiabilidade excelente para ovinos.

Como foi observado, nos meses de outubro e novembro de 2002 e janeiro de 2003, fatores ambientais poderiam ter interferido na avaliação e colocado os animais em risco de infecção aguda. No entanto, como os animais se encontravam em bom estado nutricional e livre de outras causas de anemia, o grau de correlação do OPG e Famacha foi baixo $(0,4)$. Porém, mesmo durante este período de maior desafio parasitário, 
Tabela 1 - Grau Famacha estimado, número de ovos por grama de fezes (OPG), percentual de hematócrito e evolução da avaliação os observadores em duas ocasiões, em caprinos.

\begin{tabular}{lcccc}
\hline Animal & Famacha estimado & OPG* & Hematócrito & $\begin{array}{c}\text { Avaliação** } \\
\text { 21/maio }-05 / \text { julho }\end{array}$ \\
\hline 1 & $21 /$ maio $-05 /$ julho & $21 /$ maio $-05 /$ julho & $21 /$ maio $-05 /$ julho & $0-0$ \\
2 & $2-3$ & Neg -2100 & $26-22$ & $1-7$ \\
3 & $2-1$ & $100-200$ & $28-21$ & $0-0$ \\
4 & $2-2$ & $300-600$ & $25-26$ & $6-4$ \\
5 & $3-3$ & Neg - Neg & $28-26$ & $0-0$ \\
6 & $1-1$ & $400-600$ & $32-28$ & $6-3$ \\
7 & $3-1$ & Neg -2700 & $28-25$ & $2-4$ \\
8 & $2-3$ & Neg -500 & $29-26$ & $5-0$ \\
9 & $2-1$ & Neg - Neg & $32-29$ & $11-1$ \\
10 & $3-2$ & Neg -300 & $32-28$ & $2-1$ \\
11 & $2-2$ & Neg - Neg & $29-28$ & $7-2$ \\
& $3-1$ & Neg -1100 & $29-26$ & $3,5-1,64$ \\
\hline
\end{tabular}

* Todas as fêmeas apresentavam-se em avançado estado de gestação.

** A pontuação é conferida comparando-se os valores estimados da tabela do guia Famacha e os valores obtidos de hematócrito. Os valores foram calculados a partir da média de três examinadores.

somente $28 \%$ dos animais apresentaram OPG acima do limite estabelecido. Neste caso, sugerese que, nas estações de maior risco para cada região, o rebanho seja avaliado semanalmente, visando assim evitar queda na produção e possíveis mortes.

VATTA et al. (2001) determinaram que a aplicação do método Famacha para caprinos deve considerar alguns critérios específicos. Como foi determinada neste experimento, a correlação entre os valores de hematócrito e a coloração da conjuntiva nesta espécie, pode apresentar diferença significativa em relação aos dados de OPG. A avaliação clínica determinou que alguns fatores podem comprometer a condição sanitária do rebanho, como o estado nutricional e a condição reprodutiva. Estes fatores são importantes também para ovinos.

A funcionalidade do método Famacha foi avaliada através de um questionário para acadêmicos de Medicina Veterinária posteriormente ao treinamento teórico-prático. A análise revelou que $89 \%$ dos entrevistados consideram que os benefícios deste guia estão entre muito bom e excelente, comparado com $3 \%$ que opinaram como ruim e fraco. A praticidade foi definida como sendo muito boa e excelente por $77 \%$ dos entrevistados, comparada com $10 \%$ que a definiram como ruim e fraca. Foi manifestada a intenção de que a orientação técnica deve ser oferecida através de "dias de campo" (MOLENTO \& TASCA, 2002). A preparação dos observadores através de cursos é

Tabela 2.- Evolução da pontuação dos avaliadores em cinco exames em ovinos.

\begin{tabular}{llll}
\hline Data & No. de animais & Pontuação* & Comentários \\
\hline $20-10-2001$ & 37 & 5,69 & Grande variação na coloração e a pouca experiência dos examinadores \\
$10-11-2001$ & 37 & 3,6 & Oito animais discrepantes \\
$24-11-2001$ & 37 & 2,6 & Oito animais discrepantes \\
$07-12-2001$ & 37 & 0,9 & No. pequeno de animais dos graus 3, 4 e 5 \\
$22-12-2001$ & 37 & 0,24 & No. pequeno de animais dos graus 3, 4 e 5 \\
\hline
\end{tabular}

* A pontuação é conferida comparando-se os valores estimados da tabela do guia Famacha e os valores obtidos de hematócrito. Os valores foram calculados a partir da média de três examinadores. 
fundamental para que o profissional esteja apto a observar as variações na coloração entre os diferentes graus, assim como para diminuir as variações entre avaliadores. $\mathrm{O}$ avaliador também deve observar que o estresse, a subnutrição e outros fatores infecciosos podem causar anemia ou hiperemia, e o profissional não pode negligenciar o tratamento em um animal clinicamente suspeito.

\section{CONCLUSÕES}

Foi possível testar este método e comprovar sua aplicabilidade tanto em ovinos como em caprinos adultos, como alternativa no controle do H. contortus. A redução nos custos de produção, a diminuição no volume de substâncias químicas lançadas ao meio ambiente e o auxílio na tomada de decisão quanto ao tratamento dos animais fazem do método Famacha um sistema atraente. Os dados apresentados refletem um período crítico do ano caracterizado por uma alta taxa de contaminação das pastagens e desafio parasitário na região Noroeste do Estado do Paraná, semelhante a outras regiões de produção pecuária no Brasil.

Muito embora a eficácia do composto químico utilizado neste estudo se encontra no limite para a resistência anti-helmíntica, devido ao baixo grau do intervalo de confiança $(85 \%)$, novos estudos devem ser conduzidos para determinar o efeito da utilização do método Famacha na manutenção da eficácia das drogas. A hipótese é que a estratégia de tratamento seletivo através da utilização do método Famacha causará uma diminuição na pressão de seleção da população de parasitas (adultos e larvas em refugia), com conseqüente redução do processo de resistência parasitária.

Sugere-se que este método seja utilizado juntamente com outras técnicas quando da adoção de um sistema integrado de manejo parasitário, visando a manutenção da eficácia dos compostos químicos por períodos prolongados.

\section{AGRADECIMENTOS}

Este estudo foi financiado pela Universidade Paranaense, Instituto de Pesquisa e Ambiência Científica, Unipar. Protocolo n ${ }^{\circ} 103 / 2001$.

\section{REFERÊNCIAS BIBLIOGRÁFICAS}

BATH, G.F.; VAN WYK, J.A. Using the Famacha system on commercial sheep farms in South Africa. In:
INTERNATIONAL SHEEP VETERINARY CONGRESS, 1., 1992, Cidade do Cabo, África do Sul. Anais... Cidade do Cabo: University of Pretoria, 2001. V.1. 346p. p. 3 .

ECHEVARRIA, F. et al. The prevalence of anthelmintic resistance of sheep in Southern Latin America: Brazil. Veterinary Parasitology, v.62, p.199-206, 1996.

GORDON, H.M.; WHITLOCK, H.V. A new technique for counting nematode eggs in sheep faeces. Journal of the Council of Scientific and Industrial Research, v.12, p.50-52, 1939.

MALAN, F.S.; VAN WYK, J.A. The packed cell volume and color of the conjunctivae as aids for monitoring Haemonchus contortus infestations in sheep. In: BIENNIAL NATIONAL VETERINARY CONGRESS, 1., 1992, Grahamstown, África do Sul. Anais... Grahamstown : South African Veterinary Association, 1992. V.1. p.139.

MOLENTO, M.B.; DANTAS, J.C. Validação do guia Famacha para diagnóstico clínico de parasitoses em pequenos ruminantes no Brasil: resultados preliminares. In: ENCONTRO INTERNACIONAL DE AGROECOLOGIA E DESENVOLVIMENTO SUSTENTÁVEL. 1., 2001. Botucatu, SP. Anais... Botucatu : Universidade Estadual de São Paulo, 2001. V.1, p.58.

MOLENTO, M.B.; PRICHARD, R.K. Nematode control and the possible development of anthelmintic resistance. Revista Brasileira de Parasitologia Veterinária, v.8, p.75-86, 1999.

MOLENTO, M.B.; TASCA, C. Opinião de acadêmicos em Medicina Veterinária sobre a utilização do guia Famacha como método de controle parasitário. In: CONGRESSO BRASILEIRO DE ESPECIALIDADES EM MEDICINA VETERINÁRIA, 2002, Curitiba, Paraná. Anais... Curitiba : Sociedade Paranaense de Medicina Veterinária, 2002. p. 156.

ROBERTS, J.; SWAN, R.A. Quantitative studies of ovine haemonchosis. The interpretation and diagnostic significance of the changes in serial egg counts of Haemonchus contortus in a sheep flock. Veterinary Parasitology, v.9, p.211216,1982

VAN WYK, J.A.; MALAN, F.S.; BATH, G.F. Rampant anthelmintic resistance in sheep in South Africa - what are the options? In: WORKSHOP OF MANAGING ANTHELMINTIC RESISTANCE IN ENDOPARASITES, 1997, Sun City, South Africa. Proceedings... Sun City, 1997. p.51-63.

VAN WYK, J.A. et al. Anthelmintic resistance in South Africa: surveys indicate an extremely serious situation in sheep and goat farming. Onderstepoort Journal of Veterinary Research, v.66, p.273-284, 1999.

VATTA, A. et al. Testing for clinical anemia caused by Haemonchus spp. in goats under resource-poor conditions in South Africa using an eye colour chart developed for sheep. Veterinary Parasitology, v.99, p.1-14, 2001. 
VIEIRA, L.S.; CAVALCANTE, A.C.R. Anthelmintic resistance in goat herds in the State of Ceará. Pesquisa Veterinária Brasileira, v.19, p.99-103, 1999.
WERTEJUK, M. On the invasive larvae of the gastrointestinal nematodes of sheep and their identification. Acta Parasitologica Polonica, v.2, p.235-256, 1955. 